\title{
Structure Of Flame Balls At Low Lewis-number (SOFBALL): Preliminary Results from the STS-83 and STS-94 Space Flight Experiments
}

\author{
Paul D. Ronney, Ming-Shin Wu and Howard G. Pearlman \\ Department of Aerospace and Mechanical Engineering \\ University of Southern California, Los Angeles, CA 90089 \\ Karen J. Weiland \\ Microgravity Science Division \\ NASA Lewis Research Center, Cleveland, $\mathrm{OH} 44135$
}

\begin{abstract}
Results from the Structure Of Flame Balls At Low Lewis-number (SOFBALL) space flight experiment conducted on the MSL-1 Space Shuttle missions are reported. Several new insights were obtained, including: much lower buoyancy-induced drift speed than anticipated pre-flight; repulsion of adjacent flame balls due to their mutual interaction; remarkable sensitivity of flame balls to small accelerations resulting from Orbiter attitude control maneuvers; and very similar net heat release for all flame balls in all mixtures tested. Comparison of experimental results to computational predictions reveals limitations in current models of $\mathrm{H}_{2}-\mathrm{O}_{2}$ chemistry for very lean mixtures. It is discussed how the results of these space experiments may provide an improved understanding of the interactions of the two most important phenomena in combusting materials, namely chemical reaction and transport processes, in the unequivocally simplest possible configuration.
\end{abstract}

\section{INTRODUCTION}

Flames are typically classified as "premixed" flames, where all reactants (for example fuel and air) are intimately mixed on the molecular level before the combustion process is started, and "nonpremixed" or "diffusion" flames, where the fuel and oxidant must mix before combustion can take place. Premixed flames include the familiar laboratory Bunsen burner as well as the flames inside of a gasoline-fueled internal combustion engine. It is well known that premixed gas flames containing too little fuel ("lean mixtures") or too much fuel ("rich mixtures") will not burn. Despite many years of study, these lean and rich "flammability limits" and the behavior of weakly burning flames near these limits are not well understood. For example, the best available predictions of the burning velocities of very lean hydrogen-air mixtures near flammability limits are higher than the experimental measurements by a factor of 2 (Ref. 1). Away from these limits, the agreement between model and experiment is much more satisfactory. Understanding combustion under lean conditions is critical to the design of efficient, clean-burning combustion engines. Leanburning hydrogen-fueled engines are frequently considered as a means of meeting California's upcoming ultralow emission vehicle standards and the proposed federal emission standards for beyond the year 2000 . Also, knowledge of near-limit behavior of flames is necessary for the assessment of fire and explosion hazards in mine shafts, oil refineries and chemical plants ${ }^{2}$.

It has been known for many years ${ }^{2}$ that most near-limit phenomena are influenced by gravity through the effects of buoyant convection on the transport rates of thermal energy and reactants to/from the chemical reaction zones. This has motivated a number of recent experiments on flame propagation in a $\mu \mathrm{g}$ environment ${ }^{3.4}$. It has been found that in a $\mu \mathrm{g}$ environment the absence of buoyant convection emphasizes other transport mechanisms, including the unequal rates of diffusion of thermal energy and diffusion of molecular reactants (the Lewis number effect) and the spectral radiation emitted from the gaseous combustion products. As a consequence of the change in the relative magnitudes of various transport mechanisms at $\mu \mathrm{g}$, a number of new near-limit phenomena have been observed. Perhaps the most unusual of these are "flame balls," which are the subject of the SOFBALL flight experiment.

\section{SCIENTIFIC BACKGROUND}

Over 50 years ago, Zeldovich ${ }^{5}$ shorved that the steady heat and mass conservation equations admit a solution corresponding to a stationary spherical flame or "flame ball" (Fig. 1), just as the same governing 
equations in planar geometry admit a steadily propagating flame as a solution for every mixture. In the former case the solutions are characterized by a radius (r.) and in the latter case by the burning velocity. The mass conservation equation in a steady spherically symmetric system with no sources or sinks, $\nabla \cdot(\rho \mathbf{u})=0$, where $\rho$ is the density and $\mathbf{u}$ the fluid velocity vector, requires that $\mathbf{u}$ be identically zero everywhere. In spherical geometry, the solution to steady, convection free diffusion equations for temperature and chemical species, $\nabla^{2} \mathrm{~T}=0$ and $\nabla^{2} Y$, where $\mathrm{T}$ is the temperature and $\mathrm{Y}$ the fuel mass fraction, are of the form $c_{1}+c_{2} / r$, where $r$ is the radial coordinate and $c_{1}$ and $c_{2}$ are constants. This form satisfies the requirement that $T$ and $Y$ be bounded as $r \rightarrow \infty$. For cylindrical and planar geometry the corresponding forms are $c_{1}+c_{2} \ln (r)$ and $c_{1}+c_{2} r$, respectively, which are obviously unbounded as $r \rightarrow \infty$. For this reason theory admits steady flame ball solutions, but not "flame cylinder" or "flame slab" solutions. Zeldovich showed that for an adiabatic flame ball, the energy and species conservation equations could be combined to infer the temperature at the surface of the flame ball (T.):

$$
\mathrm{T}_{*}=\mathrm{T}_{\infty}+\left(\mathrm{T}_{\mathrm{ad}}-\mathrm{T}_{\infty}\right) / \mathrm{Le}
$$

where $T_{\infty}$ is the ambient temperature, $T_{a d}$ the adiabatic flame temperature and Le the Lewis number, defined as ratio of the mixture thermal diffusivity $(\alpha)$ to the mass diffusivity (D) of the stoichiometrically limiting reactant (which is always the fuel for the purposes of the discussion here.) Thus, the temperature profile is given by $T(r)=T_{\infty}+\left(T .-T_{\infty}\right) r / r$, where $r$ is the flame ball radius. Zeldovich also showed that flame ball solutions are unstable and thus probably would not be physically observable, just as planar flames are frequently subject to instabilities which prevent them from remaining planar.

Forty years later after Zeldovich's analysis, seemingly stable flame balls were accidentally discovered in drop-tower experiments ${ }^{6}$ in lean hydrogen-air mixtures (Le $\left.\approx 0.3\right)$ and subsequently observed in drop-tower and aircraft experiments ${ }^{7}$ in $\mathrm{H}_{2}-\mathrm{O}_{2}-\mathrm{CO}_{2}(\mathrm{Le} \approx 0.2), \mathrm{H}_{2}-\mathrm{O}_{2}-\mathrm{SF}_{6}(\mathrm{Le} \approx 0.06)$ and $\mathrm{CH}_{4}-\mathrm{O}_{2}-\mathrm{SF}_{6}$ mixtures $(\mathrm{Le} \approx 0.3$ ). The $\mu \mathrm{g}$ environment of the drop tower was needed to obtain spherical symmetry and to avoid buoyancy-induced extinction of the flame balls. The following sequence of phenomena was observed as the mixtures were progressively weakened by addition of air, inert gas or chemical inhibitor. For mixtures sufficiently far from flammability limits, flame balls consistently split into more flame balls, resulting in an expanding spherical front composed of many individual cells, similar to the cellular fronts resulting from the diffusive-thermal instability widely observed at $1 \mathrm{~g}$ in mixtures with low Le. For weaker mixtures closer to the flammability limits, stable flame balls were observed. For still weaker mixtures all flames extinguished. It was concluded that flame balls would probably occur in all combustible mixtures with low Le for mixtures close to the extinction limits, however, the short duration of drop tower experiments and the substantial fluctuations in the acceleration level in aircraft $\mu \mathrm{g}$ experiments precluded definite conclusions.

These results were found to be qualitatively the same over the range $0.06<\mathrm{Le}<0.3$, with $\mathrm{H}_{2}$ and $\mathrm{CH}_{4}$ fuels, with or without added $\mathrm{CF}_{3} \mathrm{Br}$ (a chemical inhibitor) and at pressures from 0.5 to $3 \mathrm{~atm}$, indicating that variations in Lewis number over this range, chemical mechanisms, and radiation spectra do not qualitatively influence these phenomena.

The apparent discovery of stable flame balls motivated a search for a stabilizing mechanism. Zeldovich ${ }^{5}$ had noted the possibility of heat losses stabilizing flame balls. The effects of volumetric radiative losses (e.g., due to gas radiation) on flame balls were analyzed by Buckmaster and collaborators ${ }^{8,9}$. When the heat losses are not too strong, two stationary flame ball radii are predicted (Fig. 2), a "large" flame ball that is strongly affected by heat loss and a "small" flame ball that is nearly adiabatic, and when the losses are sufficiently strong no solutions exist, indicating a flammability limit. As the limit is approached, the difference between the radii of the "large" and "small" balls decreases to zero. Stability analyses ${ }^{8.9}$ showed that all small flame balls are unstable to radial disturbances, i.e., the flame will either grow outward from the equilibrium radius (and possibly develop into a propagating flame) or collapse inward and extinguish. Large flame balls with weak heat loss effects, i.e., far from the flammability limits, are predicted ${ }^{8,9}$ unstable to three-dimensional disturbances, which is consistent with the observation of splitting cellular flames in these mixtures. Consequently, a portion of the large flame branch close to the extinction limits is stable to both types of disturbances, which is consistent with the experimental observations.

It has also been predicted ${ }^{10}$ that stable flame balls can only exist for mixtures with mixtures having Le less than a critical value which is less than unity, which explains why flame balls are not observed for 
mixtures with Le less than but close to unity (e.g. $\mathrm{CH}_{4}$-air) or larger than unity (e.g. $\mathrm{C}_{3} \mathrm{H}_{8}$-air), even for nearlimit mixtures at $\mu \mathrm{g}$. Instead, conventional propagating flames are observed under these conditions.

Flame balls have several unique and interesting properties which indicate a number of practical applications. Since they are one-dimensional, steady and convection-free, they are the simplest possible type of premixed flame structure and therefore provide a useful test-bed for theoretical and numerical models of the interaction between chemical and transport processes in flames, especially near flammability limits. Some of these interactions are not predicted well even by the best currently available models. For example, numerical simulations of flame ball properties ${ }^{11,13}$ employing detailed chemical, radiation and transport models in a spherically symmetric system show that different published chemical reaction models for hydrogen-oxygen oxidation predict widely varying flame ball characteristics (Fig. 3), even though all of these models can accurately predict the burning velocities of flames in hydrogen-air mixtures farther away from the extinction limits (Fig. 4). This is particularly significant because models of hydrocarbon combustion chemistry must have an accurate $\mathrm{H}_{2}-\mathrm{O}_{2}$ sub-mechanism if they are to be able to model hydrocarbons accurately. Also, since flame balls can be observed in mixtures that are well outside the conventionally defined extinction limits, microgravity can be a more hazardous environment from the point of view of fire safety. Flame balls warrant particular concern because they do not propagate; this makes fire detection and suppression more difficult. This potential problem is compounded because hydrogen burns without visible radiation or smoke, and because sources of hydrogen abound on spacecraft (e.g., in propulsion and fuel cell systems). Flame balls may also be relevant to the turbulent combustion of mixtures with low Lewis number because flame balls are more robust than plane flames (the computed ${ }^{11}$ radiation-induced extinction limit of flame balls in lean $\mathrm{H}_{2}$-air mixtures is $3.43 \% \mathrm{H}_{2}$, whereas for plane flames it is $11.1 \%$.) Consequently, sufficiently strong turbulence may extinguish planar flames, whereas flame balls could persist under the same conditions. Hence, structures reminiscent of flame balls could be the prevalent ones in near-limit turbulent combustion of lean hydrogen-air mixtures in engines.

\section{EXPERIMENTAL APPROACH}

Based on the above discussion, the objectives of the SOFBALL experiment can be summarized as follows:

- Determine whether steady, stationary flame balls can exist in an extended-duration $\mu \mathrm{g}$ environment

- Assess the influence of gaseous radiation on flame ball size and stability

- Determine whether flame ball motion (if observed) is due to the non-zero gravity level (present to a small extent even in the Spacelab environment)

- Determine the effect of Lewis number and radiation on flame balls through the use of mixtures employing different diluent gases

The drop-tower and aircraft $\mu \mathrm{g}$ experiments indicate that a very long duration and high quality $\mu \mathrm{g}$ environment is necessary to assess the steady properties and stability limits of flame balls. A theoretical estimate of the time required can be made in the following way. The response time of flame balls is on the order of the time for thermal diffusion of energy from the near-field region of the flame ball to the far-field region. Theory 8,9 shows that the former region is characterized by radii of the order of $r$. and the latter region is characterized by radii of the order of $\theta r_{*}$, where $\theta \equiv E / R_{*}$ is the non-dimensional activation energy, $E$ the dimensional overall activation energy of the heat release reactions and $R$ the gas constant. Consequently, the far-field time scale is of the order $(\theta \mathrm{r} .)^{2} / \alpha$. Since typical values of $\mathrm{r} ., \theta$ and $\alpha$ are $5 \mathrm{~mm}, 10$ and $20 \mathrm{~mm}^{2} / \mathrm{s}$, respectively, for lean $\mathrm{H}_{2}$-air mixtures, a representative time scale for flame ball evolution is $125 \mathrm{~s}$ - much longer than the time available from drop-tower or aircraft facilities. This evolution time scale is confirmed by numerical simulations ${ }^{11,13}$. Another consideration is that the gravity level must be small enough that the flame balls are not significantly affected by convection. The drift velocity of flame balls based on aircraft $\mu \mathrm{g}$ data was found $\mathrm{d}^{7}$ to be $1.5(\mathrm{gr} .)^{1 / 2}$, where $\mathrm{g}$ is the gravitational acceleration. Since velocities on the order of $\alpha / \mathrm{r}$. are sufficient to disturb flame balls ${ }^{12}, \mathrm{~g}<1.5 \times 10^{-4} \mathrm{go}_{\mathrm{o}^{\prime}}$, where $\mathrm{g}_{\mathrm{o}}$ is earth gravity, is required to obtain diffusion-dominated flame balls (as opposed to convection-dominated flames.) To insure that the conductive flux, represented by $\alpha / r_{\text {. }}$, is significantly less than the convective flux, represented by the drift velocity, the acceleration level should be a factor of $\theta$ less than this, or $1.5 \times 10^{-5} \mathrm{go}_{\mathrm{o}}$. Another requirement is that the acceleration is small enough that the flame balls do not drift into the walls 
of the combustion chamber before at least one characteristic evolution time has elapsed. This coincidentally also requires a gravity level of $1.5 \times 10^{-5} \mathrm{~g}$, or lower in the combustion chamber employed, which has a radius of $160 \mathrm{~mm}$. This required time and quality of $\mu \mathrm{g}$ indicate the need for space experiments. The SOFBALL experiment on MSL-1 provided the requisite $\mu \mathrm{g}$ environment.

The SOFBALL experiments were performed in the Combustion Module-1 facility, developed by the NASA-Lewis Research Center in Cleveland, Ohio. A cylindrical chamber of $320 \mathrm{~mm}$ inside diameter and $320 \mathrm{~mm}$ length was filled from one of 14 bottles containing a pre-specified weakly combustible $\mathrm{H}_{2}$-air, $\mathrm{H}_{2}-\mathrm{O}_{2}-$ $\mathrm{CO}_{2}$ or $\mathrm{H}_{2}-\mathrm{O}_{2}-\mathrm{SF}_{6}$ gas mixture and ignited using electric sparks of variable deposited energy up to $700 \mathrm{~mJ}$ with spark gaps variable from 0.35 to $10 \mathrm{~mm}$. The optimal energy and gap were determined pre-flight by aircraft $\mu \mathrm{g}$ tests. The flame balls evolving from this ignition source were observed using two intensified video cameras (sensitive to visible and near-IR emissions from 400 to $900 \mathrm{~nm}$ ) with orthogonal views, a set of six thermocouples to measure gas temperature, and four radiometers (two unfiltered and two with a $5 \mu \mathrm{m}-7.5$ $\mu \mathrm{m}$ band-pass filter to detect only $\mathrm{H}_{2} \mathrm{O}$ radiation) to measure the radiant heat flux emitted from the flames. Additionally, the chamber pressure was recorded during the test and the pre- and post-combustion gas compositions were measured using a gas chromatograph. The on-orbit acceleration levels were measured by the four different on-board accelerometer systems (OARE, MMA, SAMS and QSAM). The PI Microgravity Services (PIMS) team provided the SOFBALL team with OARE acceleration data transformed to the combustion chamber location; the accelerations reported below use these specially processed data.

\section{EXPERIMENTAL RESULTS}

Two of the fifteen scheduled SOFBALL experiments were successfully conducted during the shortened STS-83 mission. A total of seventeen test points were performed on STS-94, including all fifteen planned combustion tests plus two additional tests (obtained by creating gas mixtures from bottle residuals), of which sixteen of these seventeen mixtures ignited. These mixtures tested on STS-83 and STS-94 produced from one to nine flame balls, with the mixtures having more fuel producing multiple flame balls. Most of the tests burned for 500 seconds, until the experiment timed out and a mixing fan extinguished the flames. As discussed later, it had not been expected that any flame balls would last this long. Unfortunately, it was not possible to change the experiment duration after the STS- 83 mission but before the STS-94 mission, mostly due to the time that would have been required to re-verify the control software. Ten of the mixtures from STS-94 were ignited a second time (since there was ample remaining fuel in many cases) and eight of these burned for an additional 500 seconds.

A typical image of the flame balls is observed in $\mathrm{H}_{2}$-air mixtures is shown in Fig. 5. For this and most of the $\mathrm{H}_{2}$-air tests, unlike most $\mathrm{H}_{2}-\mathrm{O}_{2}-\mathrm{CO}_{2}$ and $\mathrm{H}_{2}-\mathrm{O}_{2}-\mathrm{SF}_{6}$ tests, all flame balls extinguished before the $500 \mathrm{~s}$ experiment time-out. This is mainly because the flame balls are larger in $\mathrm{H}_{2}$-air mixtures and the fuel (hydrogen) diffusivity is higher in $\mathrm{N}_{2}$ than in $\mathrm{CO}_{2}$ or $\mathrm{SF}_{6}$. Both of these factors led to more volume of fuel being consumed per unit time in $\mathrm{H}_{2}$-air tests for the same number of flame balls.

Figure 6 shows measured flame ball radii for $\mathrm{H}_{2}$-air mixtures as a function of the fuel concentration. The radius is arbitrarily defined as the half-width of the flame ball intensity profile and one-third of the maximum intensity. Data obtained in the space experiments is very consistent with data obtained in the 10second drop tower at the Japan Microgravity Center (JAMIC) ${ }^{21}$ as well as recent aircraft ${ }^{21}$ tests. (Other properties, notably temperature profiles and radiative emission, are very different in the drop tower, aircraft and space experiments.) The flame ball radii are in poor agreement with our computational predictions, mainly because of uncertainties in the chemical reaction rates (Fig. 3), and in fact none of the mechanisms predict radii in agreement with experimental observations.

A typical image of the flame balls is observed in $\mathrm{H}_{2}-\mathrm{O}_{2}-\mathrm{CO}_{2}$ mixtures is shown in Fig. 7. The flame balls drifted several tens of $\mathrm{mm}$ away from each other during this test, but the imaginary point at the "center of mass" of the three balls hardly moved at all during the $500 \mathrm{~s}$ test period. Thus, the flame ball movement is likely due to an interaction of the balls rather than buoyancy-induced drift. Both of these mechanisms of flame ball drift are discussed in the following section.

Figure 8 shows measured flame ball radii for $\mathrm{H}_{2}-\mathrm{O}_{2}-\mathrm{CO}_{2}$ mixtures as a function of the fuel concentration. Again, data obtained in the space experiments is very consistent with data obtained in the JAMIC and aircraft tests. Note that our numerical model ${ }^{13}$ predicts that no mixtures below $5.8 \% \mathrm{H}_{2}$ are flammable, and even for $5.8 \% \mathrm{H}_{2}$ and higher, the predicted flame ball radii are much smaller than those observed in drop tower and aircraft $\mu$ g experiments. The discrepancies are believed to be due to the effect of 
reabsorption of emitted radiation by the $\mathrm{CO}_{2}$ diluent gas, which is neglected in current models ${ }^{11,13}$ Reabsorption is expected to be important for the $\mathrm{CO}_{2}$-diluted mixture because the Planck mean absorption length $\left(\mathrm{L}_{\mathrm{P}}\right)$ for $\mathrm{CO}_{2}$ at $1 \mathrm{~atm}$ and $300 \mathrm{~K}$ is $42 \mathrm{~mm}$, which is much smaller than the radius of the combustion chamber. These effects are also important for the $\mathrm{H}_{2}-\mathrm{O}_{2}-\mathrm{SF}_{6}$ mixtures $\left(\mathrm{L}_{\mathrm{P}} \approx 3.5 \mathrm{~mm}\right)$ but not for $\mathrm{H}_{2}$-air mixtures, where the only radiating specie is the product $\mathrm{H}_{2} \mathrm{O}$ where $\mathrm{L}_{\mathrm{l}} \approx 1 \mathrm{~m}$. Consequently, for $\mathrm{H}_{2}-\mathrm{O}_{2}-\mathrm{CO}_{2}$ and $\mathrm{H}_{2}-\mathrm{O}_{2}-\mathrm{SF}_{6}$ mixtures much of the radiation emitted from within or near the flame ball will not be lost to the chamber walls but instead will be reabsorbed within the gas. An approximate theoretical model of flame balls including radiative reabsorption effects ${ }^{14}$ predicts that as the absorption length scale decreases, the flame ball size increases and the flammability limit shifts to weaker mixtures, which is consistent with the observed discrepancies. A computational estimate of an upper bound for reabsorption effects in $\mathrm{H}_{2}-\mathrm{O}_{2}-$ $\mathrm{CO}_{2}$ mixtures was obtained ${ }^{13}$ by artificially neglecting $\mathrm{CO}_{2}$ radiation entirely in the numerical model, which is equivalent to assuming zero absorption length. Figure 8 shows that the actual flame ball radii are much closer to that predicted assuming this upper bound for reabsorption effects rather than that predicted without reabsorption.

The gas temperature data obtained from the $\mathrm{H}_{2}$-air test shown in Fig. 5 are given in Fig. 9, and the chamber pressure and radiometer data are given in Fig. 10. These data are much more dynamic than that from the previous test, which is expected since 5 balls of varying size were observed and all extinguished at varying times during the test. The maximum temperature observed was $576^{\circ} \mathrm{C}$, which compares to the maximum predicted ${ }^{13}$ temperature of $862^{\circ} \mathrm{C}$. This discrepancy is not surprising considering that no flame ball was observed to make a "direct hit" on a thermocouple junction.

\section{DISCUSSION}

\section{Buoyancy-induced flame ball drift}

It had not been expected that the flame balls would last more than about half of the $500 \mathrm{~s}$ experiment time-out period because the drift velocity $(v)$ of flame balls was expected to be given by the formula ${ }^{7}$

$$
\mathrm{v} \approx 1.5 \sqrt{g r_{*}}
$$

This empirical relation was inferred from data on drift velocity obtained in $\mathrm{KC}-135 \mu \mathrm{g}$ aircraft experiments, where accelerations are on the order of $10^{-2} \mathrm{~g}_{0}$. According to Eq. (2), even at $1 \mu \mathrm{g}$, a flame ball with radius 3 $\mathrm{mm}$ will drift $129 \mathrm{~mm}$, nearly the radius of the chamber, in $500 \mathrm{~s}$. The form of Eq. (2), v $\sim \sqrt{g r_{*}}$, is that of a bubble rising in an inviscid fluid ${ }^{15}$, which implies viscosity effects are negligible. This is reasonable for the conditions in the aircraft experiments, where 50 is a typical Reynolds number (Re) based on the observed ginduced drift velocity and the "equivalent buoyant radius" of the flame ball, inferred to be about five times the visible radius ${ }^{7}$. (The high-temperature, low density region of the flame ball extends far beyond the visible radius, as implied by Fig. 1, thus the volume of buoyant gas is much larger than the volume of the flame ball itself.) In contrast, at $1 \mu \mathrm{g}$, Re will be much less than unity and the viscous, creeping-flow relation for bubbles ${ }^{15}$

$$
\mathrm{v}=\frac{1}{3} \frac{g r_{b}^{2}}{v_{o}}\left(\frac{\rho_{b}}{\rho_{o}}-1\right) \frac{\mu_{o}+\mu_{b}}{\mu_{o}+1.5 \mu_{b}}
$$

where the b subscript refers to the bubble properties, should be employed instead of Eq. (2). Modeling the flame ball as a bubble whose radius is the equivalent buoyant radius $(=5 \mathrm{r}$.) mentioned above and using temperature-averaging of gas properties based on a maximum temperature of $1200 \mathrm{~K}$, Eq. (3) becomes

$$
\mathrm{v}=2.4 \frac{g r_{*}^{2}}{v^{\prime}}
$$


While the validity of Eq. (4) has not yet been confirmed, this appears to be an example of circumstances where ground-based $\mu \mathrm{g}$ experiments led to inaccurate predictions of the behavior of a space experiment.

With very low drift velocities, it is possible that in many cases the flame balls could continue to bum until their fuel supply has been depleted sufficiently that the remaining mixture was no longer flammable, rather than drifting into the chamber wall first. In a few of the Estimates for the maximum possible burn duration developed based on mechanism suggest that in the $3 \mathrm{~atm} \mathrm{H}_{2}-\mathrm{O}_{2}-\mathrm{SF}_{6}$ mixtures which produced only one flame ball, the ball could burn for about 17 hours!

\section{Flame ball mutual repulsion}

It was found that in both tests the flame balls drifted apart from each other throughout the duration of the bum. This had been seen in drop tower tests, but the test durations had been too short to obtain meaningful data on separation rates. In the space experiments it was found that the drift rate continually decreased with time. Figure 11 shows the mean separation between the three flame balls seen in Fig. 7 as a function of time, and the mean radius of separation, determined by finding the radius of the circle passing through all three flame ball centers. The camera view (not shown) orthogonal to that seen in Fig. 7 shows three practically collinear balls, which indicates that in the view shown in Fig. 7, the plane of the flame balls is orthogonal to the axis of the camera lens, which in turn indicates that the measure of flame spacing seen in this view is a reasonably accurate indication of the true spacing.

A proposed mechanism of flame ball drift based on the mutual depletion of total enthalpy (chemical plus thermal) by two flame balls in the region between them is presented here. When two flame balls are in close proximity, they have two influences on each other: first, they deplete each other of reactants from the region between them (decreased chemical enthalpy) and two, they increase the temperature in the region between them (increased thermal enthalpy). Because of the enthalpy gradient, one side of the flame ball will have a temperature slightly greater than $\mathrm{T}$. while the other will be slightly lower, thus leading to differences in heat release rate on the two sides of the ball. It is proposed that the ball must drift in the direction of increasing total enthalpy at a rate whereby the convective transport of enthalpy to the ball balances this difference in heat release rate. A model of flame ball drift has been developed, resulting in the prediction ${ }^{16}$

$$
S=\left[\frac{9 \alpha_{o} r_{*} t}{1-L e}\right]^{1 / 3}
$$

The prediction of Eq. (5) with the representative parameters for Test $\# 1$ (T* $\approx 1200 \mathrm{~K}, \mathrm{~T}_{\mathrm{o}}=300 \mathrm{~K}, \alpha_{\mathrm{o}} \approx 10$ $\left.\mathrm{mm}^{2} / \mathrm{s}, \mathrm{r} .=3 \mathrm{~mm}, \mathrm{Le}=0.2\right)$ are shown in Fig. 11 , where the formula has been multiplied to by $(8 / \sqrt{3})^{1 / 3}$ to account for the additional gradient caused by presence of three rather than two flame balls as well as transformation of the prediction from mean spacing to mean radius of separation. The agreement between theory and experiment is fairly close, and so may indicate some validity for the proposed mechanism.

The flame balls were found to be much more sensitive to Orbiter Vernier Reaction Control System (VRCS) thruster firings than expected pre-flight. These firings produced a noticeable change in the flame ball position, drift speed, and especially radiometer data (Fig. 12). The strong effect of microgravity disturbances on radiation is probably due to the fact that the visible flame ball is surrounded by a much larger volume of hot but non-reacting gas. Most of the radiation is emitted from this large gas volume rather than from within the flame ball itself ${ }^{11}$. This large ball of gas is extremely susceptible to buoyancy-induced motion resulting from even the smallest VRCS impulses (on the order of $50 \mu \mathrm{g}$ for $1 \mathrm{sec}=0.5 \mathrm{~mm} / \mathrm{sec}$ ). (Careful inspection of the two data sets from STS-83 also showed this trend, but it was much less noticeable in these cases because both STS-83 tests produced multiple flame balls with more widely dispersed hot gas regions that, as a group, are much less affected by the VRCS firings than tests producing only one or two flame balls.) Note that Fig. 12 shows that only VRCS firings, which cause a net change in the Orbiter momentum, had any effect; vibrations resulting from crew and Orbiter systems operations, which do not affect the net momentum of the Orbiter, had practically no effect. Once the effects of the VRCS firings were seen, "free drift" (suppression of VRCS firings) was requested during the remaining experiments and was granted in many cases. When free drift could be maintained for the entire test period, the disturbances to the radiometer readings did not occur. 
Preliminary inspection of the flight data suggest that the flame balls respond ballistically to the VRCS impulses, that is, the impulse (change in velocity) imparted to the ball is the same as the acceleration impulse. This change in velocity then decays on a time scale of tens to hundreds of seconds, comparable to the viscous time scale associated with the flame ball and its surrounding hot gas field.

\section{SUMMARY AND CONCLUSIONS}

Two space-based experiments on STS-83 and 24 experiments on STS-94 have shown that flame balls are in fact stable for very long periods of time in a $\mu \mathrm{g}$ environment. The rate at which the flame balls drift due to the small acceleration levels on the spacecraft was far smaller than that expected before the mission. A modified mechanism of buoyancy-induced drift is proposed based on the difference between the flow regimes in the aircraft and space experiments (nearly inviscid flow vs. creeping flow), and a new mechanism of flame ball drift induced by mutual interaction of adjacent flame balls is proposed. Flame ball properties, especially radiative emission, were found to be strongly affected by orbiter Vernier Reaction Control System thruster firings. This may have important implications for combustion experiments to be performed on the International Space Station.

Another finding of these experiments is that the differences between computer models and experiments found based on earlier short-duration $\mu \mathrm{g}$ tests were also found in the space experiments. Different models of $\mathrm{H}_{2}-\mathrm{O}_{2}$ chemistry yield different predictions for flame ball properties, and it is expected that detailed evaluation of the results of the space experiments will help lead to the identification of the most appropriate chemical models for lean-limit combustion of hydrogen and hydrocarbon fuels.

A reflight of the SOFBALL experiment is planned for STS-107 in the fall of 2000 . Among the changes planned for SOFBALL-2 include longer test durations, since most flame balls were still buming at the end of the $500 \mathrm{~s}$ tests on STS-83 and STS-94. A third intensified video camera with a smaller field of view but greater spatial resolution will be added. A higher energy ignition system will be used to study still-leaner mixtures, particularly in the $\mathrm{H}_{2}$-air family. Optical filters will be used on some of the intensified video cameras in order to determine if the emission wavelength has an impact on the apparent flame ball sizes.

\section{PUBLICATIONS AND PRESENTATIONS}

\section{Refereed journal publications:}

Abid, M., Wu, M. S., Liu, J. B., Ronney, P. D., Ueki, M., K. Maruta, K., Kobayashi , H., Niioka, T. and VanZandt, D. M., "Experimental and Numerical Study of Flame Ball IR and UV Emissions," to appear in Combustion and Flame (1998).

Wu, M.-S., Liu, J. B. and Ronney, P. D., “Numerical Simulation of Diluent Effects on Flame Ball Structure and Dynamics," to appear in the Twenty-Seventh International Symposium on Combustion (1998).

Buckmaster, J. D. and Ronney, P. D., "Flame Ball Drift in the Presence of a Total Diffusive Heat Flux," to appear in the Twenty-Seventh International Symposium on Combustion (1998).

Ronney, P. D., "Understanding Combustion Processes Through Microgravity Research," to appear in TwentySeventh International Symposium on Combustion, Combustion Institute, 1998 (invited paper).

Ronney, P. D., "Premixed Laminar and Turbulent Flames at Microgravity," to appear in Space Forum (1998).

Ronney, P. D., Wu, M. S., Pearlman, H. G. and Weiland, K. J., "Experimental Study of Flame Balls in Space: Preliminary Results from STS-83," AIAA Journal, Vol. 36, pp. 1361-1368 (1998).

\section{Presentations:}

Wu, M.-S., Ronney, P. D., "Computation of Flame Ball Properties with Detailed Chemical and Transport Models," SIAM Numerical Combustion Conference, York, England, March 30-April 1, 1998.

Ronney, P. D., Wu, M.-S., Weiland, K. J. and Pearlman, H. G., "Structure of Flame Balls At Low Lewisnumber (SOFBALL): Preliminary Results from the STS-83 and STS-94 Space Flight Experiments," 36 th Aerospace Sciences Meeting, Reno, NV, January 12 - 15, 1988.

Buckmaster, J. D. and Ronney, P. D., "Flame Ball Drift," Fall Technical Meeting, Combustion Institute, Eastern Statés Section, Hartford, CT, October 27-29, 1997. 


\section{ACKNOWLEDGMENTS}

The authors wish to thank the CM-1 Project Managers, Mr. Roy Hager (retired) and Ms. Ann Over, the CM-1 Project Engineer, Mr. Ron Chucksa (retired) and the entire CM-1 engineering and operations team for their tremendous effort in designing, constructing, testing and operating the CM-1 facility. Angel AbbudMadrid, Mohammed Abid, Quin Blackburn and Jian-Bang Liu assisted with the data analysis during and after the missions. Special thanks is owed to the STS-83/94 crew for conducting the experiments, and the MSFC mission management team, led by Ms. Teresa Vanhooser and Dr. Michael Robinson, for coordinating experiment operations. This work was supported by NASA grants NAG3-1242, NAG3-1523 and NAG3-2124.

\section{REFERENCES}

${ }^{1}$ Eolfopoulos, F. N. and Law, C. K., "An Experimental and Computational Study of the Buming Rates of Ultra-lean to Moderately-rich $\mathrm{H}_{2} / \mathrm{O}_{2} / \mathrm{N}_{2}$ Laminar Flames with Pressure Variations," Twenty-Third Symposium (International) on Combustion, Combustion Institute, Pittsburgh, 1990, pp. 333-340.

${ }^{2}$ Coward, H., Jones, C., "Flammability Limits of Gases and Vapors," U. S. Bureau of Mines Bulletin 503, 1952.

${ }^{3}$ Ronney, P. D., "Effect of Chemistry and Transport Properties on Near-Limit Flames at Microgravity," Combustion Science and Technology Vol. 59, 1988, pp. 123-141.

${ }^{4}$ Abbud-Madrid, A. and Ronney, P. D., "Effects of Radiative and Diffusive Transport Processes on Premixed Flames Near Flammability Limits," Twenty-Third Symposium (International) on Combustion, Combustion Institute, Pittsburgh, 1990, pp. 423-431.

${ }^{5}$ Zeldovich, Ya. B., Theory of Combustion and Detonation of Gases Academy of Sciences (USSR), 1944.

${ }^{6}$ Ronney, P. D. "Near-Limit Flame Structures at Low Lewis Number," Combustion and Flame Vol. 82, 1990, pp. 1-14.

${ }^{7}$ Ronney, P. D., Whaling, K. N., Abbud-Madrid, A., Gatto, J. L. and Pisowicz, V. L., "Stationary Premixed Flames in Spherical and Cylindrical Geometries," AIAA Journal. Vol. 32, 1994, pp. 569-577.

${ }^{8}$ Buckmaster, J. D., Joulin, G. and Ronney, P. D., "Effects of Heat Loss on the Structure and Stability of Flame Balls," Combustion and Flame, Vol. 79, 1990, pp. 381-392.

${ }^{9}$ Buckmaster, J. D., Joulin, G. and Ronney, P. D., "Structure and Stability of Non-adiabatic Flame Balls: II. Effects of far-field losses," Combustion and Flame, Vol. 84, 1991, pp. 411-422.

${ }^{10}$ Lee, C. and Buckmaster, J. D., "The Structure and Stability of Flame Balls: a Near-Equidiffusional Flame Analysis," SIAM Journal on Applied Mathematics, Vol. 51, 1991, pp. 1315-1326.

${ }^{11}$ Wu, M. S., Ronney, P. D., Colantonio, R. and VanZandt, D., "Detailed Numerical Simulation of Flame Ball Structure and Dynamics," to appear in Combustion and Flame (1998).

${ }^{12}$ Buckmaster, J. D. and Joulin, G., "Flame Balls Stabilized by Suspension in Fluid with a Steady Linear Ambient Velocity Distribution," Lournal of Fluid Mechanics, Vol. 227, 1991, pp. 407-427.

${ }^{13} \mathrm{Wu}, \mathrm{M}$. S. and Ronney, P. D., Twenty-Seventh Symposium (International) on Combustion, Combustion Institute, Pittsburgh, 1998, to appear.

${ }^{14}$ Lozinski, D., Buckmaster, J. D., Ronney, P. D., "Absolute Flammability Limits and Flame Balls in Optically Thick Mixtures," Combustion and Flame, Vol. 97, 1994, pp. 301-316.

${ }^{15}$ Batchelor, G. K., An Introduction to Fluid Dynamics, Cambridge University Press, Cambridge, U.K., 1967.

${ }^{16}$ Buckmaster, J. D. and Ronney, P. D., "Flame Ball Drift in the Presence of a Total Diffusive Heat Flux," Twenty-Seventh Symposium (International) on Combustion, Combustion Institute, 1998, to appear.

${ }^{17}$ Frenklach, M., et al., "An Optimized Kinetics Model for Natural Gas Combustion." Twenty-Fifth Symposium (International) on Combustion, Poster 26, Session 3, 1994.

${ }^{18}$ Peters, N. in: Reduced Kinetic Mechanisms for Applications in Combustion Systems, N. Peters and B. Rogg (Eds.), Springer-Verlag, Berlin-Heidelberg, 1993, Chapters 1 and 5.

${ }^{19}$ Yetter, R. A., Dryer, F. L. and Rabitz, H., "A Comprehensive Reaction Mechanism For Carbon Monoxide/Hydrogen/Oxygen Kinetics," Combustion Science and Technology, Vol. 79, 1991, pp. 97-128.

${ }^{21}$ Mauss, F., Peters, N., Rogg, B. and Williams, F. A., in: Reduced Kinetic Mechanisms for Applications in Combustion Systems, N. Peters and B. Rogg (Eds.), Springer-Verlag, Berlin-Heidelberg, 1993, Chapter 3.

${ }^{21}$ Abid, M., Wu, M. S., Liu, J. B., Ronney, P. D., Ueki, M., K. Maruta, K., Kobayashi , H., Niioka, T. and VanZandt, D. M., "Experimental and Numerical Study of Flame Ball IR and UV Emissions," to appear in Combustion and Flame (1998). 


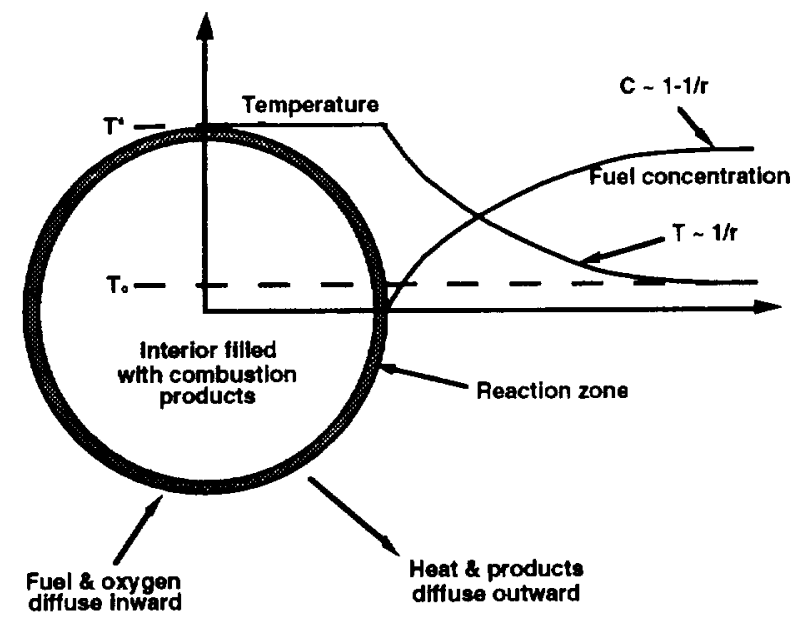

Figure 1. Schematic diagram of a flame ball, illustrated for the case of fuel-limited combustion at the reaction zone. The oxygen profile is similar to the fuel profile except its concentration is nonzero in the interior of the ball. The combustion product profile is identical to the temperature profile except for a scale factor.

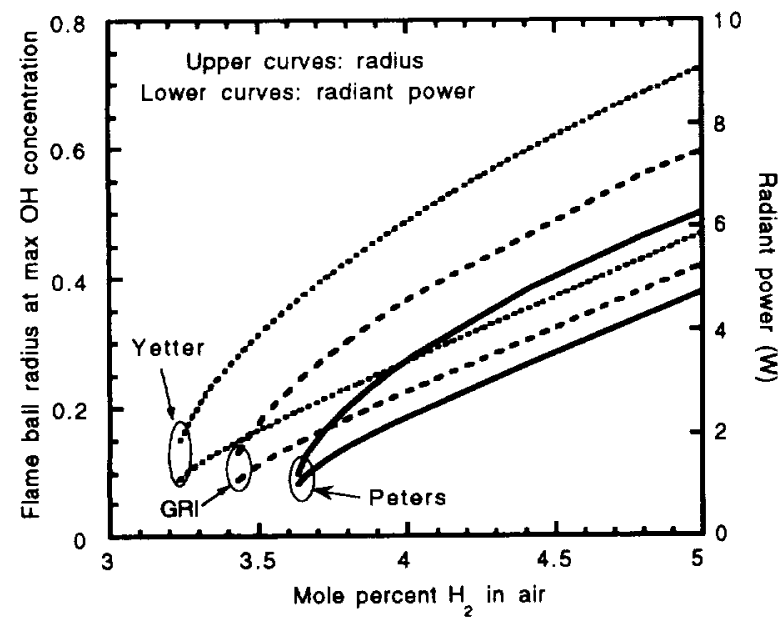

Figure 3. Predicted ${ }^{13}$ steady flame ball radii and radiant emission in $\mathrm{H}_{2}$-air mixtures using the $\mathrm{GRI}^{17}$, Peters ${ }^{18}$ and Yetter ${ }^{19}$ chemical models.

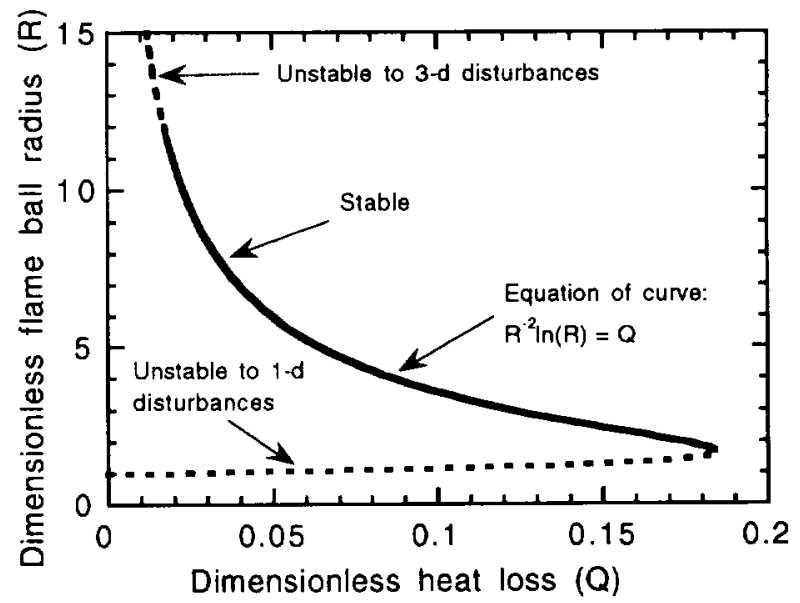

Figure 2. Theoretical prediction ${ }^{8}$ of the effect of heat loss on flame ball radius and stability properties with single-step Arrhenius chemistry, constant thermodynamic and transport properties and volumetric heat loss from the interior of the ball.

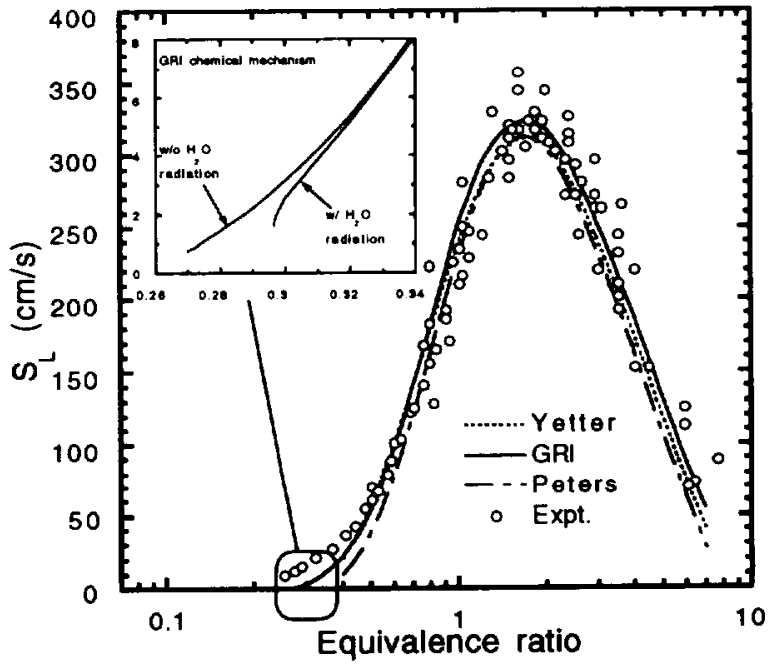

Figure 4. Predicted ${ }^{13}$ steady burning velocities $\left(S_{L}\right)$ of $\mathrm{H}_{2}$-air mixtures using the $\mathrm{GRI}^{17}$, Peters ${ }^{18}$ and Yetter $^{19}$ chemical models. A compilation ${ }^{20}$ of experimental results from several sounces is also shown. 


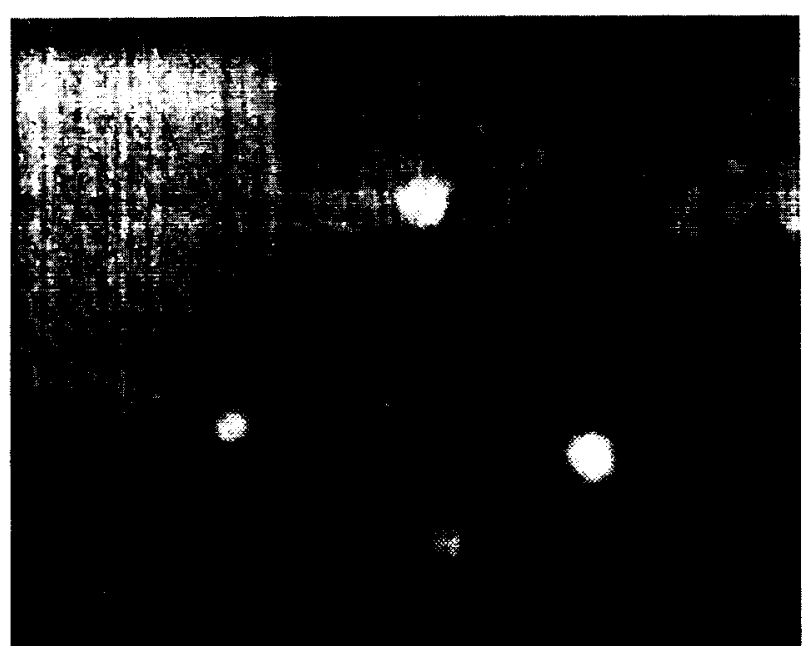

Figure 5 . Image of flame balls obtained in $3.85 \%$ $\mathrm{H}_{2} / 96.15 \%$ air mixture, taken $25 \mathrm{~s}$ after ignition. Field of view is $112 \mathrm{~mm} \times 150 \mathrm{~mm}$.

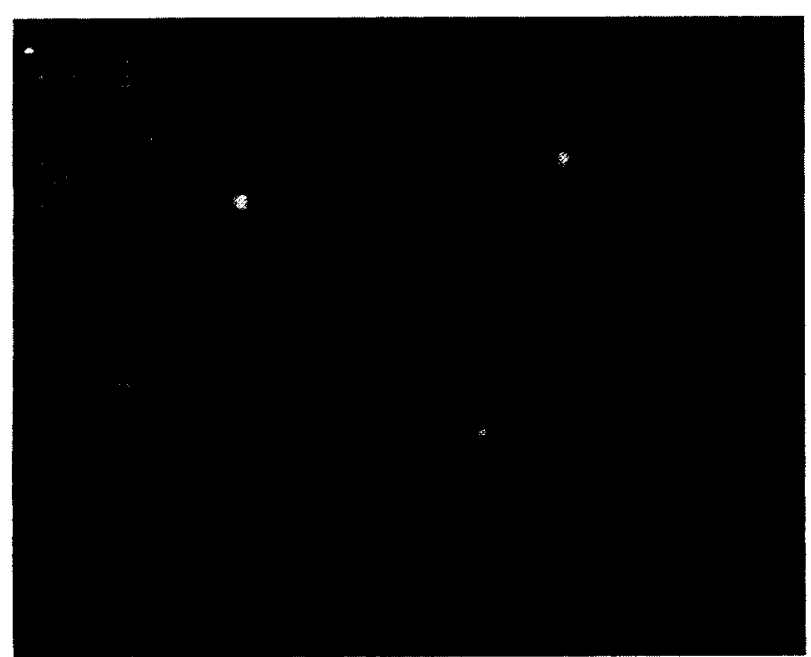

Figure 7. Image of flame obtained in $4.9 \%, \mathrm{H}_{2} /$ $9.8 \% \mathrm{O}_{2} / 85.3 \% \mathrm{CO}_{2}$ mixture, taken $130 \mathrm{~s}$ after ignition. Field of view is $112 \mathrm{~mm} \times 150 \mathrm{~mm}$.

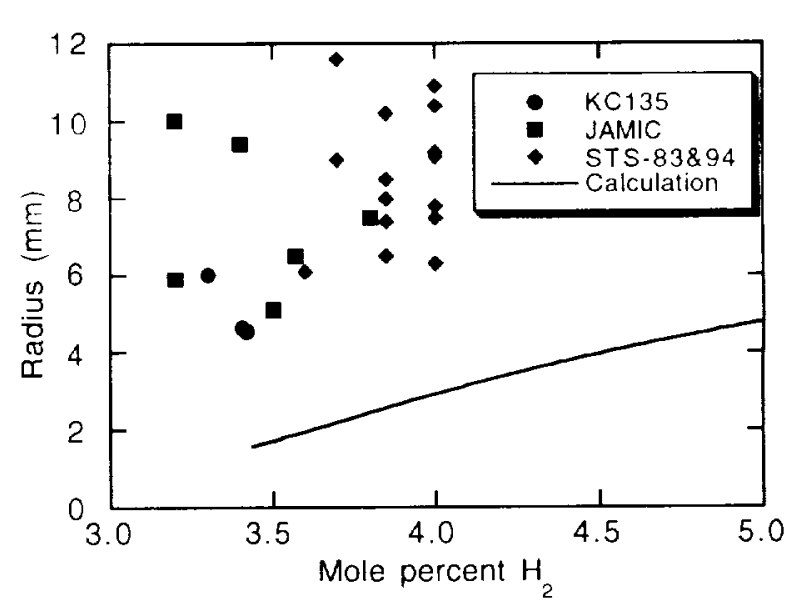

Figure 6. Predicted ${ }^{13}$ flame ball radii in $\mathrm{H}_{2}$-air mixtures along with measured flame ball radii from aircraft $\mu \mathrm{g}$ experiments ${ }^{21}$, JAMIC drop-tower experiments $^{21}$, and the STS-83/94 space experiments.

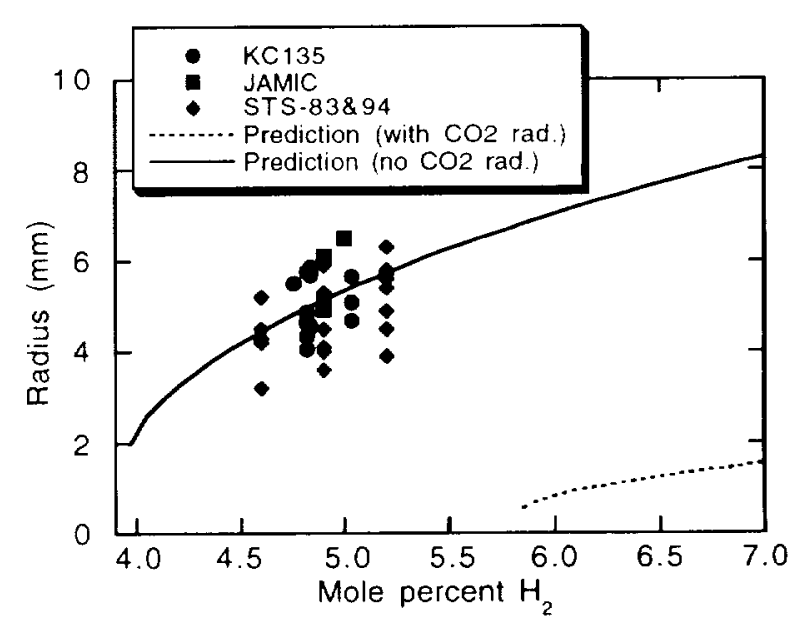

Figure 8. Predicted ${ }^{13}$ flame ball radii in $\mathrm{H}_{2}-\mathrm{O}_{2}-$ $\mathrm{CO}_{2}$ mixtures $\left(\mathrm{H}_{2}: \mathrm{O}_{2}=1: 2\right)$ including and excluding $\mathrm{CO}_{2}$ radiation, along with measured flame ball radii from aircraft $\mu \mathrm{g}$ experiments ${ }^{21}$, JAMIC droptower experiments ${ }^{21}$, and the STS-83/94 space experiments. 


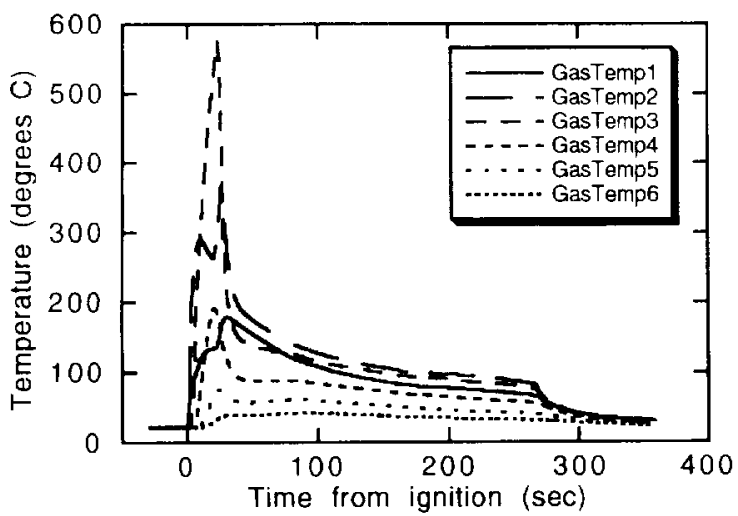

Figure 9. Measured gas temperatures for Test \#2. Thermocouple locations corresponding to GasTemp1 through GasTemp6 are 30, 50, 70, 90, 110 and $130 \mathrm{~mm}$ from spark gap, respectively.

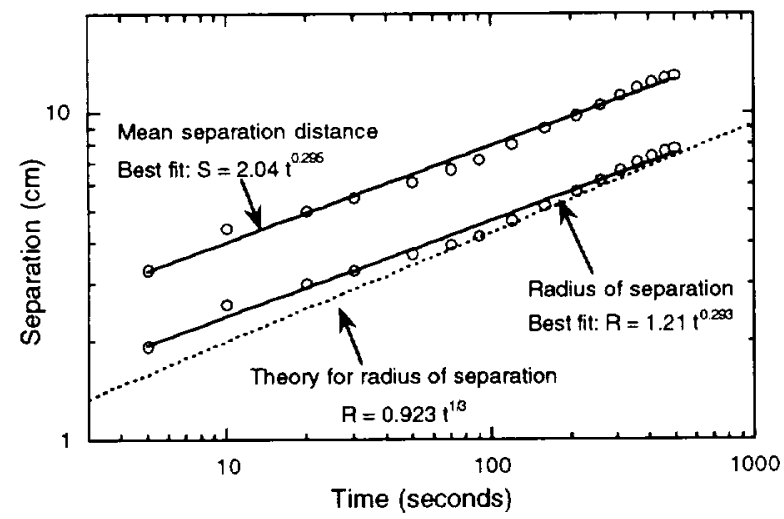

Figure 11. Observed radius of separation and mean separation of the three flame balls seen in Fig. 7 as a function of time, and comparison to theoretical predictions ${ }^{16}$.

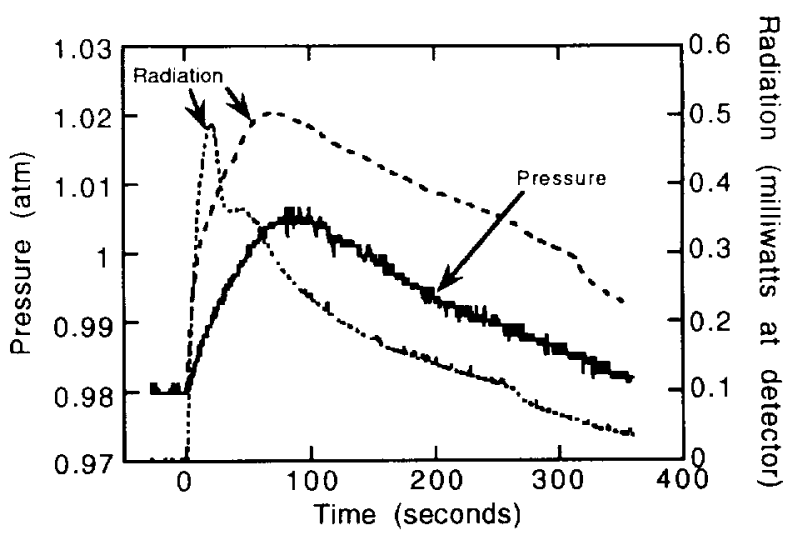

Figure 10. Measured chamber pressure and radiant emissions for Test \#2.

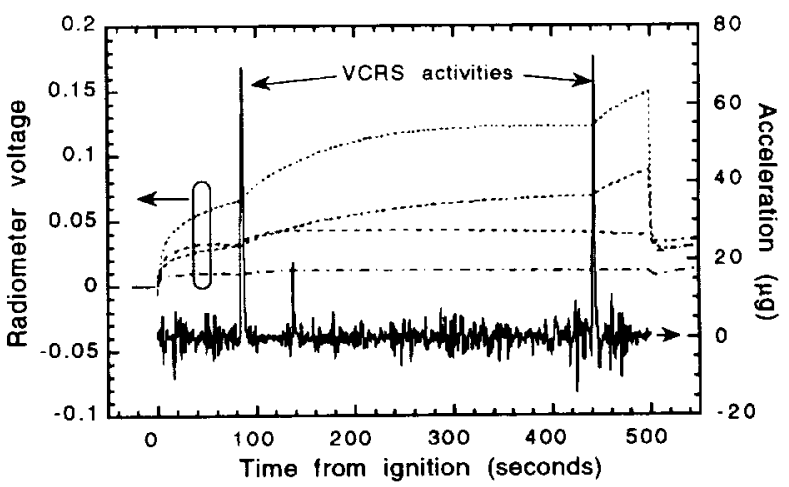

Figure 12. Example correlation of radiometer readings with Orbiter acceleration environment during STS-94 mission showing impact of VRCS thruster firings. For clarity, only the Orbiter zaxis (vertical) acceleration component is shown. Mixture: $6.50 \% \mathrm{H}_{2}-13.00 \% \mathrm{O}_{2}-80.50 \% \mathrm{SF}_{6}, 1 \mathrm{~atm}$ initial pressure. 


\title{
Structure Of Flame Balls At Low Lewis-number (SOFBALL): Results from space flight experiments on STS-83 and STS-94
}

\author{
Paul D. Ronney, Principal Investigator \\ University of Southern California, Los Angeles, CA
}

The objective of the Structure Of Flame Balls At Low Lewis-number (SOFBALL) experiment is to study the behavior and properties of a newly discovered premixed-gas flame phenomena called "flame balls." These spherical, stable, stationary flame structures, observed only in microgravity, provide the opportunity to study the interactions of the two most important processes necessary for combustion (chemical reaction and heat/mass transport) in the simplest possible configuration. The previously unobtainable experimental data collected during the STS-83 and STS-94 missions provide a comparison with models of flame stability and flame propagation limits that are crucial both for fire safety assessment on earth and in orbiting spacecraft as well as for the design of lean-burn combustion engines which provide improved fuel economy and lower pollutant formation.

The tests were conducted by filling a 26-liter chamber with a weakly combustible gas (hydrogen and oxygen highly diluted with an inert gas) and igniting the mixture with a spark. The flames were imaged using four video cameras. Radiometers, thermocouples and pressure transducers were used to deternine the heat release from the flame balls. The experiments were performed in the Combustion Module-1 facility on the Microgravity Science Laboratory (MSL-1) Spacelab missions.

Two successful tests (out of two attempts) were conducted on STS-83 and 16 successful tests (out of 17 attempts) were conducted on STS-94. Most of the tests burned for $500 \mathrm{sec}$, until the experiment timeout extinguished the flames. Over 3 hours of test data were collected.

\section{SOFBALL highlights:}

- First premixed gas combustion experiment ever performed in space

- Weakest flames ever burned, either on the ground or in space. Flame ball powers as low as one watt were measured. By comparison, a birthday candle releases about 50 watts.

- Longest-lived gas flames ever burned in space (many mixtures burned for the entire $500 \mathrm{sec}$ test duration and were still burning at the timeout.)

- Conclusive evidence of limitation of existing models of lean hydrogen-oxygen combustion chemistry, which predict many ground-based experimental results accurately but yield conflicting predictions of flame ball properties, none of which agree with the experiments. Improved models of lean combustion are currently being developed based on the SOFBALL results.

- First conclusive demonstration of the effect of reabsorption of emitted radiation on the burning characteristics of a flame, either on the ground or in space. This factor is insignificant for most laboratory-scale experiments, but of key importance in practical flames at high pressure (as in automotive and jet engines) or at large scale (as in industrial boilers).

- First conclusive demonstration of the impact of accelerations caused by Orbiter vernier thruster firings and water dumps on science data obtained during a shuttle flight.

Work supported by the SOFBALL project has led to 27 journal papers and 7 invited conference presentations by the PI and his associates since SOFBALL was approved for flight in 1991. 